\title{
La reforma de la legislación audiovisual y de la cobertura electoral en España
}

\author{
The reform of the audiovisual legislation and electoral \\ coverage in Spain
}

Francisco Campos-Freire. Universidad de Santiago de Compostela. España. francisco.campos@usc.es

$[\mathrm{CV}]$ (C)

Marta Rodríguez-Castro. Universidad de Santiago de Compostela. España. m.rodriguez.castro@usc.es

$[\underline{\mathrm{CV}}]$ (1) $\mathrm{G}$

Alejandro Gesto-Louro. Universidad de Santiago de Compostela. España. alejandrogesto@gmail.com

$[\underline{\mathrm{CV}}]$ (c)

Esta comunicación forma parte de las actividades del proyecto de investigación (RTI2018-096065-B-I00) del Programa Estatal de I $+D+I$ orientado a los Retos de la Sociedad del Ministerio de Ciencia, Innovación y Universidades (MCIU), Agencia Estatal de Investigación (AEI) y del Fondo Europeo de Desarrollo Regional (FEDER) sobre "Nuevos valores, gobernanza, financiación y servicios audiovisuales públicos para la sociedad de Internet: contrastes europeos y españoles". La autora Marta Rodríguez-Castro es beneficiaria del Programa de Formación del Profesorado Universitario (FPU16/05234) financiado por el Ministerio de Educación, Cultura y Deporte (Gobierno de España).

\author{
Cómo citar este artículo / Referencia normalizada \\ Campos-Freire, F., Rodríguez-Castro, M. y Gesto-Louro, A. (2020). La reforma de la legislación \\ audiovisual y de la cobertura electoral en España. Revista Latina de Comunicación Social, (76), \\ 143-161. https://www.doi.org/10.4185/RLCS-2020-1441
}

\section{RESUMEN}

Introducción: El objeto de este artículo es plantear la reforma de la regulación vigente en España sobre la cobertura y tratamiento de la información en los medios audiovisuales durante las campañas electorales, en relación con la obligada transposición a la legislación española, antes de finales de 2020, de la Directiva 1808/2018 de comunicación audiovisual. Metodología: Para ello, se realiza una revisión de la legislación más relevante en el campo, incluyendo la Ley Orgánica de Régimen Electoral (LOREG) de 1985, las distintas leyes de creación de los medios públicos y privados, la Ley 7/2010 General del Audiovisual (LGA), la Directiva europea de servicios audiovisuales aprobada en 2018 y la regulación en otros países europeos sobre la organización de debates electorales y campañas de desinformación. Resultados: Pese a que los 35 años de vigencia de la LOREG y su jurisprudencia condicionan el marco legal de la cobertura audiovisual de las campañas electorales, es posible incluir en la transposición y reforma de la LGA 7/2010 la regulación de los debates y nuevas medidas sobre protección del pluralismo, independencia y verificación frente a las fake news, cuestiones que un grupo de académicos españoles plantearon con motivo de la consulta pública de la Secretaría de Estado para el Avance Digital (SEAD) del Ministerio de Economía y Empresa (MEE) sobre la transposición de la Directiva 1808/2018. 
PALABRAS CLAVE: Directiva europea de servicios audiovisuales; LOREG; cobertura de la información electoral; debates electorales; desinformación.

\begin{abstract}
Introduction: The purpose of this article is to propose the reform of the regulation in force in Spain on the coverage and treatment of information in the audiovisual media during electoral campaigns, in relation to the mandatory transposition into Spanish legislation, before the end of 2020, of Directive 1808/2018 of audiovisual communication. Methodology: For this, a review of the most relevant legislation in the field is carried out, including the Organic Law of Electoral Regime (LOREG by its acronym in Spanish) of 1985, the different laws of creation of public and private media, General 7/2010 Law of Audiovisual (LGA by its acronym in Spanish), the European Audiovisual Services Directive approved in 2018 and the regulation in other European countries on the organization of electoral debates and disinformation campaigns. Results: Although the 35 years of validity of the LOREG and its jurisprudence condition the legal framework of the audiovisual coverage of the electoral campaigns, it is possible to include in the transposition and reform of the LGA 7/2010 the regulation of the debates and new measures on the protection of pluralism, independence, and verification against fake news, issues that a group of Spanish academics raised during the public consultation of the Secretary of State for Digital Advancement (SEAD by its acronym in Spanish) of the Ministry of Economy and Business (MEE by its acronym in Spanish) on the transposition of Directive 1808/2018.
\end{abstract}

KEYWORDS: Audiovisual Media Services Directive; LOREG; electoral information coverage; electoral debates; disinformation.

\title{
CONTENIDOS
}

1. Introducción. 2. Legislación y cobertura electoral. 3. Cobertura informativa electoral. 4. Organización y regulación de debates. 5. Nueva regulación audiovisual y electoral. 6. Conclusiones. 7. Referencias bibliográficas.

\section{Introducción}

En el mes de noviembre de 2018 se aprobó la nueva Directiva europea 1808/2018 de prestación de servicios de comunicación audiovisual, que revisa la anterior normativa del mismo rango de $2010 \mathrm{y}$ que obliga a los Estados a adaptar su legislación sobre comunicación audiovisual. En el caso de España, afecta a la legislación básica establecida por la Ley 7/2010 General del Audiovisual (LGA) y a las leyes de las Comunidades Autónomas con competencias en la materia. El plazo para la transposición de la Directiva de 2018 a la legislación española finaliza en el último trimestre de 2020.

La Directiva de 2018 tiene como objetivo principal la regulación de las plataformas digitales, nuevos operadores del ecosistema mediático que hasta la fecha solo estaban bajo la normativa legal del comercio electrónico, provocando competencia comercial asimétrica y gran impacto en los modelos de negocio de los medios tradicionales (Campos-Freire, De Aguilera y Rodríguez-Castro, 2018).

La Ley 7/2010 General del Audiovisual, aprobada en la legislatura del presidente socialista José Luis Rodríguez Zapatero, es la normativa básica surgida en el contexto del preludio de una importante crisis económica en España y de la fuerte influencia de dos operadores comerciales (Atresmedia y Mediaset) que se consolidan como duopolio estable del sector (Mani Redondo, 2017). En materia de cobertura electoral audiovisual, especialmente en lo que se refiere a los medios de comunicación públicos, la legislación básica audiovisual española de 2010 se limita al dictado de la Ley Orgánica 
de Régimen Electoral (LOREG). En este aspecto siguen la misma línea las leyes de los medios públicos (Ley 17/2006 de 5 de junio de la Radio y la Televisión de titularidad estatal, RTVE) del Estado y de las Comunidades Autónomas.

El marco de la regulación electoral en España, en sus diversos aspectos generales y en los relacionados con la comunicación en particular, está desarrollado a través de la Ley Orgánica 5/1985, de 19 de junio, del Régimen Electoral (LOREG), que ha experimentado en los últimos 35 años una veintena de modificaciones puntuales, pero no una reforma en profundidad, en línea con lo que ha cambiado la sociedad $\mathrm{y}$, especialmente, la estructura y funcionamiento de los medios de comunicación. Tampoco las leyes de comunicación audiovisual han desarrollado ni han adaptado los principios básicos de la LOREG a los cambios tecnológicos, de producción y consumo de la información.

A lo establecido en la LOREG, especialmente en los artículos 53 al 69, se remite toda la legislación audiovisual producida y modificada en las cuatro últimas décadas, desde las primeras leyes de la televisión pública (estatal y autonómica) y privada, hasta la recopilación y actualización de toda esta regulación en la Ley 7/2010 General de Comunicación Audiovisual. El ámbito de la comunicación en la regulación electoral afecta también a la legislación sobre la publicidad, particularmente a la publicidad institucional, y a la Ley Orgánica de Protección de Datos Personales y Garantía de los Derechos Digitales (LOPD).

Varias de esas cuestiones han cambiado en el transcurso de las últimas décadas como consecuencia de la profunda metamorfosis que ha sufrido el ecosistema mediático, el impacto de las plataformas digitales globales (Miguel de Bustos e Izquierdo Castillo, 2019) y las nuevas formas de consumo o uso de la comunicación. La naturaleza e importancia de esos cambios está constatada en la nueva Directiva europea 2018/1808 por la que se modifica la Directiva 2010/13/UE sobre la coordinación de determinadas disposiciones legales, reglamentarias y administrativas de los Estados miembros relativas a la prestación de servicios de comunicación audiovisual, que fue aprobada el 14 de noviembre del año pasado y publicada en Diario Oficial de la Unión Europea de 28 del mismo mes.

En el preámbulo de la Directiva de 2018 se reconoce que desde 1989

el mercado de los servicios de comunicación audiovisual ha evolucionado de forma significativa y rápida, debido a la convergencia actual entre la televisión y los servicios de internet. Los avances técnicos han hecho posibles nuevos tipos de servicios y experiencias de los usuarios. Los hábitos de visionado, en particular los de las generaciones más jóvenes, han cambiado significativamente. Aunque la pantalla de la televisión siga siendo un dispositivo importante para compartir experiencias audiovisuales, muchos espectadores se han decantado por otros dispositivos portátiles para ver contenidos audiovisuales. (DOUE, 2018)

Por lo tanto, el foco de esa revisión han sido esas nuevas formas de consumo y, sobre todo, los nuevos prestadores de servicios audiovisuales -las llamadas plataformas y redes digitales- para buscar una regulación más equilibrada con respecto a los operadores tradicionales, así como con respecto a la responsabilidad de los contenidos que difunden, buena parte de ellos producidos por los usuarios. 
En línea con esa disposición europea, los Estados han iniciado los procesos de reforma de sus respectivas legislaciones audiovisuales. El gobierno español, a través de la Secretaría del Estado para el Avance Digital del Ministerio de Economía y Empresa (MEE, 2019), realizó a principios de 2019 una consulta pública previa sobre la modificación de la Ley 7/2010, de 31 de marzo, General de la Comunicación Audiovisual, que se cerró el 22 de febrero y en la que se formulaban varias preguntas y cuestiones sobre los aspectos a incluir en la reforma de la legislación audiovisual española; no sólo los que pudieran derivarse de la nueva Directiva europea, sino también otros que debieran encajarse en la actualización de la citada regulación.

En dicha consulta pública participaron varias organizaciones, instituciones y profesorado de las Universidades españolas. A raíz de la invocación específica de la consulta pública, un grupo de profesores y profesoras de varias Universidades plantearon la necesidad de aprovechar la transposición de la regulación europea para realizar una profunda revisión de la Ley 7/2010 General de Comunicación Audiovisual y también de algunos aspectos relacionados con la problemática actual de la cobertura de las campañas electorales por parte de los medios audiovisuales, en particular los de servicio público. Este aspecto concreto de la cobertura de las campañas electorales y la actualización de la legislación es el que motiva esta investigación.

Al respecto se plantean las siguientes preguntas: 1. La vigencia e imprecisión de la LOREG en determinados aspectos mediáticos, ¿requiere su reforma y/o ampliación de su regulación a través de otras leyes derivadas? 2. ¿Deben ser regulados por ley los debates electorales y ser incluidos, en todo caso, entre las obligadas prestaciones que deben organizar los medios audiovisuales de comunicación públicos? 3. ¿Existe un modelo normativo europeo de regulación de los debates electorales en los medios audiovisuales? 4. ¿Hay margen legal en la transposición de una directiva europea de comunicación audiovisual para la extensión de aspectos regulatorios de la legislación de cobertura electoral? 5. La problemática de la desinformación y otras cuestiones de la actual sociedad de la información, ¿justifican el establecimiento de medidas especiales de verificación, respeto al pluralismo y de protección de la calidad democrática en la cobertura de las campañas electorales?

\section{Legislación y cobertura electoral}

El texto consolidado de la LOREG aprobada en 1985 recoge 16 artículos que abordan cuestiones relacionadas con la comunicación, propaganda y actos de cobertura de las campañas electorales. En dicho texto consolidado se recoge la legislación original de 1985 y las sucesivas modificaciones introducidas hasta 2019, que se comentan más adelante. A continuación, se enuncian y comentan los artículos específicos de la LOREG que tratan sobre la propaganda, organización, cobertura y difusión de la comunicación sobre los actos electorales.

El artículo 53 establece la prohibición y los límites de la propaganda antes y después de las campañas electorales, salvo en lo que se refiere a actividades de los partidos, coaliciones y federaciones. El artículo 54 atribuye la regulación de los actos públicos de las campañas electorales a las Juntas Electorales Provinciales, reservando las facultades de orden público a las autoridades gubernativas. Los artículos 55, 56 y 57 determinan las obligaciones y disposiciones que corresponden a los ayuntamientos para facilitar lugares y medios a los partidos, asociaciones, coaliciones o federaciones y candidaturas para realizar actividades de propaganda electoral.

La redacción de estos artículos apunta al uso tradicional de los espacios fijos estáticos (murales) para la colocación de los típicos carteles electorales, pero no precisa, en cambio, si en esos lugares se pudieran instalar - por ejemplo- pantallas o medios interactivos electrónicos que permitiesen realizar y geolocalizar interacciones con la ciudadanía. La tecnología lo permite, pero la legislación no lo 
contempla, aunque tampoco lo prohíbe expresamente. En este caso estaríamos ante una situación de vacío legal o de regulación como actividad de un nuevo medio de comunicación.

El artículo 58 establece el derecho a contratar inserciones de publicidad en la prensa periódica, radio "y en cualquier otro medio de difusión privado", pero sin dar a lugar a que exista discriminación en su admisión. Está prohibida la comunicación comercial televisiva de naturaleza política, como establece la Ley 7/2010 General del Audiovisual (LGA). La redacción de los artículos correspondientes de la LOREG y de la LGA de 2010, respectivamente, son ambiguos hoy en día con respecto a los conceptos de publicidad, comunicación comercial y diferenciación de los medios de difusión, y no contemplan, en lo que se refiere a la comunicación y difusión electoral, dos nuevas realidades del ecosistema mediático: la convergencia y los nuevos medios derivados de Internet.

Una nueva redacción y codificación de la regulación electoral exigiría, en lo que se refiere a la actividad de la comunicación, contemplar la nueva estructura y especificidades de los medios de difusión en la sociedad de Internet, así como los aspectos sociales convergentes que las Directivas, legislaciones o debates actuales que contemplan cuestiones de dignidad humana, igualdad, no discriminación por razón de sexo, raza, etnia, nacionalidad, religión, discapacidad, orientación sexual, derechos digitales, fake news, etc.; valores tradicionales y nuevos que deberían estar a resguardo de la comunicación en las campañas electorales, sin que ello suponga colisionar con la libertad de expresión y opinión.

La sección sexta de la LOREG, a través de los artículos 59 a 67, establece las condiciones de utilización de los medios de comunicación de titularidad pública en las campañas electorales. Se refieren dichos artículos a las tarifas especiales para los envíos postales de propaganda electoral, al derecho a espacios gratuitos de propaganda en las emisoras de televisión y radio de titularidad pública, la forma de distribución de dichos espacios gratuitos, los baremos de proporcionalidad fijados y las Comisiones de Radio y Televisión de las Juntas Electorales encargadas de repartir las asignaciones de los tiempos establecidos.

El artículo 62 señala que

si el ámbito territorial del medio o el de su programación fueran más limitados que el de la

elección convocada, la distribución de espacios se hace atendiendo al número total de votos

que obtuvo cada partido, federación o coalición en las circunscripciones comprendidas en el

correspondiente ámbito de difusión o, en su caso, de programación. (LOREG, 1985)

En el caso de elecciones generales (artículo 63) se toman como referencia los resultados de los anteriores comicios al Congreso de los Diputados, criterio que prevalece en el caso de coincidir con votaciones autonómicas y municipales: si coinciden autonómicas y locales prevalece el primero de estos criterios y los programas regionales de los medios nacionales, y si se celebran solo comicios para renovación de los ayuntamientos, los resultados anteriores de ese ámbito son el marco de referencia.

El artículo 64, objeto de la mayoría de las reclamaciones ante las Juntas Electorales por parte de las nuevas candidaturas, marca la distribución del tiempo de los espacios gratuitos en los medios de comunicación públicos. El baremo establecido por la LOREG es el siguiente:

a) Diez minutos para los partidos, federaciones y coaliciones que no concurrieron o no obtuvieron representación en las anteriores elecciones equivalentes o para aquellos que, 
habiéndola obtenido, no hubieran alcanzado el 5 por 100 del total de votos válidos emitidos en el territorio nacional o, en su caso, en las circunscripciones a que hace referencia el artículo 62.

b) Veinte minutos para los partidos, federaciones y coaliciones que, habiendo obtenido representación en las anteriores elecciones equivalentes, hubieran alcanzado entre el 5 y el 20 por 100 del total de votos a que se hace referencia en el párrafo a).

c) Treinta minutos para los partidos, federaciones y coaliciones que, habiendo obtenido representación en las anteriores elecciones equivalentes, hubieran alcanzado, al menos, un 20 por 100 del total de votos a que hace referencia el apartado a). (LOREG, 1985)

Añade dicho artículo 64 que el derecho a los tiempos de emisión gratuita enumerados en el apartado anterior sólo corresponde a aquellos partidos, federaciones o coaliciones que presenten candidaturas en más del 75 por 100 de las circunscripciones comprendidas en el ámbito de difusión o, en su caso, de programación del medio correspondiente. Para las elecciones municipales se estará a lo establecido en las disposiciones especiales de esta Ley. Los partidos, asociaciones, federaciones o coaliciones que no cumplan el requisito de presentación de candidaturas establecido en el apartado anterior tienen, sin embargo, derecho a diez minutos de emisión en la programación general de los medios nacionales si hubieran obtenido en las anteriores elecciones equivalentes el 20 por 100 de los votos emitidos en el ámbito de una Comunidad Autónoma en condiciones horarias similares a las que se acuerden para las emisiones de los partidos, federaciones y coaliciones. (LOREG, 1985)

Los artículos 65 a 67 marcan las competencias, entre las que se halla la distribución de los espacios gratuitos en los medios estatales, y la organización de la Junta Electoral Central a través de su Comisión de Radio y Televisión, integrada por ocho jueces y cinco representantes propuestos por los partidos políticos en el plazo de seis meses después de la constitución del Congreso de los Diputados. Si los partidos políticos no se ponen de acuerdo para la elección de sus representantes ante la respectiva Junta Electoral, como ocurrió en Cataluña en los últimos procesos electorales, dicho órgano no se puede constituir y en ese caso pasa a actuar el órgano central. La Junta Electoral Central puede delegar competencias en las Juntas autonómicas y/o provinciales, 
para los comicios de los ámbitos respectivos. En todo caso, la Junta Electoral Central es el organismo ante el que cabe recurrir decisiones de las juntas autonómicas o provinciales.

En la composición de la Comisión de Radio y Televisión de la Junta Electoral Central hasta 2019 llama la atención la ausencia de representantes con perfil de especialistas en materia de comunicación, bien sea del ámbito periodístico, audiovisual o de la publicidad. Además de los jueces que forman parte de ella, los representantes propuestos por los partidos políticos son catedráticos de Derecho o de Ciencia Política. Lo lógico sería que la regulación exigiese al menos algún representante experto en los ámbitos de la comunicación sobre los que tiene que actuar dicha comisión.

Sin cuestionar la personalidad ni los conocimientos de los académicos representados, no es fácil que por su formación puedan dominar aspectos de franjas de programación, evolución de audiencias, emisiones de todo tipo de canales, difusiones de streaming, emisiones digitales, webs, plataformas y aspectos de redes sociales. Aunque, en realidad, tampoco muchos de esos aspectos están contemplados, como debieran, en la regulación electoral vigente. Sí que consta, sin embargo, en el artículo 67, la exigencia y garantía de neutralidad, que está condicionada por esas y otras cuestiones en la realidad del ecosistema mediático actual.

Los artículos 68 y 69, correspondientes a las respectivas secciones séptima y octava, se refieren a los derechos de rectificación y a la difusión de las encuestas electorales. Las exigencias de acompañamiento de los datos técnicos de las encuestas son obvias y pertinentes, aunque la legislación debería ser más garantista para requerir -en caso de manipulaciones flagrantesdictámenes de oficio de los organismos reguladores de la comunicación para poner en evidencia tratamientos inadecuados de los datos. El impedimento de publicar encuestas cinco días antes de la votación pierde sentido frente a la dimensión global de Internet, que trasciende el espacio territorial estatal de la prohibición, como ocurre actualmente.

Entre 1987 y 2019 se realizaron 23 modificaciones de la Ley Orgánica de Régimen Electoral, pero en el ámbito de la cobertura electoral realizada por los medios de comunicación es necesaria una nueva codificación y reforma para la adaptación a la realidad mediática actual. El uso de los datos de la identidad digital y de su instrumentalización a partir de los perfiles de las plataformas y redes digitales, que provocaron el escándalo de la empresa Cambridge Analytica -creada por Robert Mercer y Steve Bannon, el polémico asesor del presidente Trump- por su utilización en las elecciones norteamericanas y en la campaña del Brexit del Reino Unido (Zunger, 2018; Schneble, Elger y Shaw, 2018; Common, 2018), es un nuevo aspecto que, junto al de las fake news, preocupa a los estados y que ha sido motivo de creación de grupos de expertos de alto nivel para la elaboración de informes para la Comisión Europea (HLEG, 2018).

España aprobó en 2018 una Ley Orgánica de Protección de Datos Personales y Garantías de los Derechos Digitales (LOPD), que introduce una controvertida modificación del artículo 58.bis de la LOREG que regula la utilización de medios tecnológicos e identidades personales en las actividades electorales. Organizaciones como ISACA (2018) cuestionan la redacción de ese artículo de la LOREG y sostienen que desprotege el uso de los datos personales, "dejando un camino abierto a la manipulación de los electores". Opinan que, al no ser considerada la propaganda electoral como un fin comercial, los ciudadanos no podrían evitar su inclusión en la lista Robinson por el acoso con ese tipo de mensajes. Por su parte, la Agencia Española de Protección de Datos (AEPD) defiende que no permitirá que los partidos políticos puedan realizar perfiles de datos ideológicos, sexuales, de religión o de cualquier otro tipo que se puedan obtener de los ciudadanos a través de las redes sociales u otros servicios. 


\section{Cobertura informativa electoral}

La programación informativa de la cobertura de las campañas electorales se establece a través de los planes elaborados por la dirección de los medios de comunicación públicos, sometidos a informe y aprobación de sus correspondientes consejos de administración, de los que se da cuenta a las respectivas juntas electorales que únicamente intervienen sobre ellos si se vulnera la obligada neutralidad que determina la LOREG en su artículo 66. Dicha programación comprende los programas de apertura y cierre de campañas, espacios de seguimiento en los informativos diarios o semanales, entrevistas y debates. La redacción del mencionado artículo alude también, en su segundo punto, a las emisoras de televisión privada.

El artículo 66 determina:

1) El respeto al pluralismo político y social, así como a la igualdad, proporcionalidad y la neutralidad informativa en la programación de los medios de comunicación de titularidad pública en período electoral, serán garantizados por la organización de dichos medios y su control previstos en las Leyes. Las decisiones de los órganos de administración de los referidos medios en el indicado periodo electoral son recurribles ante la Junta Electoral competente de conformidad con lo previsto en el artículo anterior y según el procedimiento que la Junta Electoral Central disponga.

2) Durante el periodo electoral las emisoras de titularidad privada deberán respetar los principios de pluralismo e igualdad. Asimismo, en dicho periodo, las televisiones privadas deberán respetar también los principios de proporcionalidad y neutralidad informativa en los debates y entrevistas electorales así como en la información relativa a la campaña electoral de acuerdo a las Instrucciones que, a tal efecto, elabore la Junta Electoral competente. (LOREG, 1985)

Por eso, a la hora de efectuar la programación y la asignación de la cobertura informativa, los medios audiovisuales de comunicación públicos siguen el criterio de reparto de tiempos, en línea con lo que establece la LOREG para los espacios gratuitos de propaganda electoral. No se trata de propaganda electoral, sino de información, pero el riesgo de ser denunciados por los partidos, coaliciones o federaciones por falta de proporcionalidad y neutralidad condiciona los estándares periodísticos de los medios públicos. Esto provoca quejas de los periodistas y de sus organizaciones profesionales, aunque el dilema no es fácil de resolver porque la doctrina legal y la jurisprudencial de las resoluciones de la Junta Electoral Central es totalmente conservadora en la interpretación del artículo 66 de la LOREG (Castro, 2008; VV AA, 2012; Marqués-Pascual, Fondevila-Gascón, De Uribe-Gil, Perelló-Sobrepere, 2016). 


\section{Organización y regulación de debates}

Pese a que los debates podrían presentarse como un instrumento de calidad democrática y de contraste imprescindible para la formación de una opinión política bien informada, la organización y regulación de los debates en televisión en las democracias parlamentarias europeas es una cuestión más reciente que en los Estados Unidos de América, donde se inauguran con la histórica confrontación dialéctica de 1960 entre Nixon y Kennedy. Tampoco se ha consolidado su continuidad en Europa tanto como en Estados Unidos, porque son varias las elecciones democráticas de la mayoría de los países europeos en las que no se celebran debates televisados. En Reino Unido, el país que crea la primera compañía de radiodifusión (la BBC, constituida en 1922), se organiza el primer debate de televisión en 2010 entre los tres candidatos de los partidos conservador, laborista y liberal (Anstead, 2015). El primer debate, de un total de tres, fue en la televisión comercial (ITV) y lo ganó, según las encuestas, el novato candidato del tercer partido, Nick Clegg, frente al laborista Gordon Brown y al conservador David Cameron. La controversia británica se suscitó en 2015 por el número de candidatos -participaron siete en el primer debate y cinco en el segundo, pero no el premier Cameron en ese último- y por la normativa de regulación, que estudió una comisión de House of Lords (2014).

Tras el histórico debate Nixon-Kennedy de 1960, Canadá televisa el siguiente en 1968. Alemania organizó el primer debate electoral en televisión en 1972 y Francia en 1974, pero Jacques Chirac se negó a enfrentarse en 2002 al ultraderechista Jean-Marie Le Pen. Tampoco en Alemania se volvieron a reanudar los debates hasta 2002 por la negativa de Helmuk Kohl de dar la alternativa a sus contrincantes. Australia televisa su primer debate en 1984. En Italia, en la década de los 90 del siglo XX, se inician los debates electorales de la RAI, realizados en las cadenas públicas debido a la directa relación de Silvio Berlusconi con la propiedad de Mediaset, el principal grupo audiovisual privado. En España el primer debate televisado tiene lugar el 24 de mayo de 1993 entre Felipe González y José María Aznar.

La autorregulación de la organización de los debates electorales televisados es el sistema dominante en las democracias parlamentarias occidentales. La legislación electoral establece las garantías democráticas de participación, diversidad y pluralidad, pero deja la capacidad de organización, siempre que no se vulneren las reglas básicas, a los actores del sistema político (partidos y coaliciones) y de la radiodifusión (cadenas de televisión) para acordar su celebración, número, formato y estructura de los debates.

Este sistema dominante ha sido cuestionado e interpelado en distintas ocasiones por algunos actores que se sentían excluidos o perjudicados por las pautas acordadas según ese criterio. La línea argumental de fondo de esos recursos es la apelación a la no discriminación de la diversidad pluralista y al acceso en condiciones igualitarias o proporcionales a un recurso público como es el espacio radioeléctrico utilizado por los radiodifusores, tal como ocurre como el derecho que tienen todos los partidos para colocar propaganda en determinados lugares e instalaciones de los espacios urbanos. Esta última línea argumental fue la apelada por el Partido Democrático Liberal alemán ante sus exclusiones de algunos debates electorales, que sin embargo el Tribunal Supremo de ese país no tuvo en cuenta (Maurer, 2016).

La autorregulación de la organización de los debates, que la tradición legislativa y jurisprudencial occidental ha consagrado sobre la responsabilidad de los actores del sistema de la democracia representativa, contrasta ante los impulsos actuales de fragmentación política, diversidad multipartidista y nuevas formas de participación democrática (Bachrach, Botwinick, 1992; Fung, 2006). Este contraste pone en cuestión si la organización de los debates, además de la potestad de 
los actores políticos y radiodifusores, es también un derecho de los públicos para conocer las ideas sobre las que depositará su voto de confianza.

No es hasta ahora así porque los partidos y sus candidatos, o los radiodifusores, no siempre quieren participar u organizar debates. Una estadística elaborada por Nick Anstead (2015) indica que de las 10 elecciones celebradas en Australia desde 1984 solo hubo debates en nueve; en Canadá, desde 1968 a 2015, solo hubo 10 debates de 13 elecciones; y en Alemania, desde 1972, hubo 9 debates en 12 comicios.

Los candidatos consolidados y favoritos, principalmente cuando están en el poder, son los más reacios a aceptar los debates para no incrementar el conocimiento de sus contrincantes (Garro, 2019). Pese a la a mediatización, los debates ofrecen un espacio para la confrontación y refuerzan opiniones sobre las habilidades de liderazgo, credibilidad y competencia económica si son desconocidas previamente (Babos \& Vilagi, 2018; López-García, Llorca-Abad \& Valera-Ordaz, 2018).

La influencia de los debates y de la imagen transmitida por los candidatos sobre la decisión final de los votantes son cuestiones bastante discutibles. Ganar el debate no significa ganar las elecciones. Incluso el comentado hándicap de Nixon frente a Kennedy de 1960 es rebatido por Bruschke y Divine (2017) al descubrir que los datos de la encuesta que atribuyó la desventaja estaban sesgados. Lo que sí constatan de forma unánime las investigaciones es que los debates incrementan la participación (Klein y Rosar, 2007; Gallego Reguera y Bernárdez Rodal, 2017).

Los dos grandes formatos de debates son los cara a cara (TV-Duelle), con aquellos partidos que tienen opciones a presidir el gobierno, y los del conjunto multipartidista y de la llamada "ronda de los elefantes" (Maurer \& Reinemann, 2003; Anstead, 2015). Los primeros son propios del sistema bipartidista y los segundos responden a la tendencia actual de fragmentación multipartidista de los sistemas democráticos. Las regulaciones electorales de los países europeos establecen el criterio de la proporcionalidad y la representación alcanzada en los comicios anteriores para establecer la participación, con una cuota mínima de entre el 5 y el $10 \%$ de votos o escaños a la cámara correspondiente. Es decir, una ronda de debates para los cara a cara y otra ronda para el resto de los candidatos.

La ruptura de los sistemas bipartidistas ha introducido otros formatos a tres, a cuatro y a cinco, a la vez que la ronda de los minoritarios, esta última con audiencias reducidas frente al seguimiento millonario de la de los principales líderes. Los formatos tradicionales y de la clásica matriz norteamericana se han ido diversificando y analizando con respecto a la participación directa del público, el infoentretenimiento (Conde-Vázquez, Puentes-Rivera, López-López, 2019), el desarrollo transmedia (Saavedra-Llamas, Rodríguez-Fernández, 2018), las aplicaciones de factchecking (Mazaira-Castro, Rúas-Araújo, Puentes-Rivera, 2019) y las repercusiones de las campañas a través de Internet (Tambini, 2018).

Estas últimas tendencias, que rebasan el ámbito organizativo y se adentran en la trascendencia de la estructura del ecosistema mediático, de las plataformas, la inteligencia artificial, los algoritmos y las nuevas redes de la sociedad actual de Internet, son aspectos que la nueva regulación de la comunicación también debe contemplar desde el punto de vista de la participación política y la calidad democrática. La preocupación de la Comisión europea se refleja en sus informes y en sus nuevas directivas. 
Expertos - periodistas como Manuel Campo Vidal y Alan Schroeder- y destacados políticos -como el exministro y secretario de organización del PSOE, José Blanco, y la exsecretaria de Estado de Comunicación del gobierno de Mariano Rajoy, Carmen Martínez de Castro- ponían en valor en unas jornadas celebradas en la Facultad de Ciencias Sociales y de la Comunicación de la Universidade de Vigo (Congreso Debate TV, 2019) la importancia de los debates y su regulación, en tanto en cuanto se consideran como el momento más importante de la campaña electoral (Navarro Marchante, 2019) y "un antídoto contra las fake news y la posverdad" (Campo Vidal, 2019).

\section{Nueva regulación audiovisual y electoral}

La Ley 7/2010 General del Audiovisual tiene que reformarse antes de finales de 2020 para adaptar lo dispuesto por la Directiva europea 2018/1808 de servicios audiovisuales (Perales, 2018; Díaz Arias, 2019; Instituto RTVE, 2019). Básicamente la nueva Directiva de 2018, que es una revisión ampliada de la misma norma de 2010, categoriza a las plataformas y redes sociales digitales como nuevos difusores sometidos a la regulación audiovisual, equiparando su cobertura a los condicionamientos legales de los operadores tradicionales de radio y televisión. La nueva Directiva de servicios audiovisuales amplía también otros aspectos sociales de protección de menores, contra la xenofobia, alfabetización mediática, necesidad de reguladores independientes y flexibilización de las emisiones de publicidad a través de los medios audiovisuales.

La Directiva, aprobada a mediados de noviembre de 2018, concede a los Estados 21 meses para su transposición a las legislaciones estatales y autonómicas con competencias en materia audiovisual. Por esta razón el Ministerio de Economía y Empresa (MEE) del gobierno de España lanzó en enero de 2019 una consulta pública con 25 preguntas sobre los diversos aspectos que desarrolla la Directiva 2018/1808, a la que respondieron distintas organizaciones, profesionales y ciudadanos. Doce expertos en temas audiovisuales y profesores de distintas Universidades españoles contestaron también a esa consulta pública, algunas de cuyas respuestas reproducimos a continuación para argumentar la necesidad de las reformas audiovisuales y de cobertura electoral que se sostienen en esta comunicación (Novos Medios, 2019).

Las respuestas a dicha consulta pública sobre la reforma de la Ley 7/2010 fueron suscritas por Mercedes Caridad Sebastián, catedrática de Documentación de la Universidad Carlos III de Madrid; Juan Carlos Miguel de Bustos, catedrático de Comunicación Audiovisual de la Universidad del País Vasco; Javier Marzal Felici, catedrático de Comunicación Audiovisual de la Universidad Jaume I de Castellón; Francisco Campos Freire, catedrático de Periodismo de la Universidad de Santiago de Compostela; Carmelo Garitaonandía, catedrático de Periodismo de la Universidad del País Vasco; Mercedes Medina Laverón, profesora titular de la Facultad de Comunicación de la Universidad de Navarra; Andrés Mazaira, profesor de la Universidad de Vigo; Enrique Guerrero, profesor de la Universidad de Navarra; Ana María López Cepeda, profesora de la Facultad de Periodismo de Cuenca; Fátima García López y Sara Martínez Cardama, profesoras de la Universidad Carlos III de Madrid; y Eladio Gutiérrez Montes, experto en Televisión Digital Terrestre.

Los doce expertos académicos y profesionales del audiovisual argumentan que el proceso obligado de transposición es una oportunidad para elaborar "una nueva legislación básica estatal y autonómica que recoja la transposición de la Directiva 2018/1018 UE respondiendo al mismo tiempo a esos retos tecnológicos, económicos, de armonización audiovisual, protección de derechos, cambios de consumos y de usos sociales que anticipa la tercera década de este siglo. La tarea no es fácil, pero resulta extremadamente necesaria por su cuádruple impacto: tecnológico, económico, regulatorio y social" (Novos Medios, 2019). 
Señalan también que la actual legislación básica del audiovisual se ha quedado obsoleta:

La génesis de la Ley 7/2010 General del Audiovisual se inspiró en la política audiovisual europea de la primera década del siglo XXI, la Directiva 2007/65/UE, posteriormente completada con algunas incorporaciones de la siguiente Directiva 2010/13/UE e influencias de operadores que la aprovecharon para concentrarse o reestructurarse ante la crisis económica. La ley básica audiovisual española de 2010 nació ya tardía, demasiado coyuntural, sin desarrollo posterior, incluso luego diluida en algunos aspectos socialmente capitales. Se trata de una legislación básica pensada desde la TDT y desde el mercado dual de operadores públicos y privados nacionales, regionales o locales que se ha visto superada por la disrupción tecnológica de las plataformas y las redes digitales globales, que suponen nuevas formas de uso o consumo. Los omnipresentes algoritmos transforman los mercados, los modelos de negocio y generan nuevos impactos sociales. (Novos Medios, 2019)

Junto a cuestiones básicas como el fortalecimiento de las garantías de pluralismo, independencia, financiación del servicio audiovisual público, apoyo a la producción audiovisual europea e independiente, reforma de la CNMC y creación de un regulador independiente convergente y competente en materia de contenidos audiovisuales, la respuesta de los doce académicos de las Universidades españolas incide también en contemplar las siguientes cuestiones:

- Situación, aspectos de regulación y planes de acción ante la problemática de las fake news en España.

- Situación y criterios sobre códigos, buenas prácticas, planes de acción y regulación de la protección de los menores así como de sus datos de intimidad personal, tanto en los medios tradicionales como en los contenidos generados por los usuarios en las plataformas.

- Tratamiento y regulación en el marco legal de los aspectos relacionados con racismo, xenofobia y terrorismo.

- Armonización de la legislación audiovisual estatal y autonómica así como del reconocimiento constitucional de la diversidad cultural y lingüística. 
- Criterios para el establecimiento de mecanismos plurales y participativos de representación de la diversidad de los consumidores, usuarios, ciudadanos y organizaciones empresariales, profesionales y sociales en los sistemas de gobernanza y regulación.

- Criterios para la inclusión de indicadores de pluralismo, diversidad, promoción de la igualdad y accesibilidad de personas con capacidad visual y auditiva.

- Criterios para la regulación e impulso de las acciones de alfabetización mediática.

- Criterios sobre las situaciones de dominio en el uso de datos y algoritmos por parte de las plataformas frente a los operadores tradicionales en la gestión de la publicidad programática.

- Criterios y nuevos modelos de financiación de la producción de ficción y de fomento de las industrias creativas ante la irrupción de nuevas formas de consumo y de redes de operadores de Internet.

- Criterios para el refuerzo de la independencia en los órganos de gobernanza de los servicios audiovisuales públicos y las entidades reguladoras del audiovisual.

- Criterios para una participación y representación social más activa y proactiva en los citados órganos.

- Criterios para la protección y conservación del patrimonio audiovisual. (Novos Medios, 2019)

Por último, ante la pregunta 25 de la Secretaría de Estado para el Avance Digital (SEAD) del Ministerio de Economía y Empresa sobre qué soluciones alternativas se podrían plantear en relación con otras modificaciones a introducir en el marco regulador audiovisual, se responde lo siguiente:

Como idea general, es igualmente conveniente y aconsejable el proceso de interlocución con los agentes y partes relacionadas para otras modificaciones, entre ellas, la reforma de las normas de cobertura de las campañas electorales en los medios de comunicación tradicionales y la nueva problemática surgida con los temas de desinformación y fake news en dichos procesos democráticos, como han regulado y están regulando varios países europeos y del mundo. Esta modificación trasciende a los ámbitos de la Ley Orgánica del Régimen Electoral y de las leyes audiovisuales, estatales y autonómicas. El cambio de los medios de 
comunicación y de la sociedad de la información desde que se aprobó la LOREG es importantísimo. Y por ello esta ley, sus desarrollos y resoluciones merecen una revisión y adaptación al contexto actual. (Novos Medios, 2019)

\section{Conclusiones}

La revisión de la legislación electoral y audiovisual realizada pone en evidencia que la respuesta a la primera pregunta sobre la obsolescencia e imprecisión de la LOREG requiere su revisión o desarrollo a través de sus normas audiovisuales derivadas. Porque la LOREG es una ley orgánica, los académicos y expertos concluyen que su reforma a fondo requeriría una coyuntura política de mayor estabilidad y consenso que la registrada en los últimos años y la que se vislumbra para los próximos inmediatos. No obstante, la obligada reforma de la legislación audiovisual abre una ventana de oportunidad para actualizar las cuestiones relacionadas con la cobertura electoral a través de dichos medios de comunicación.

La regulación de los debates electorales es necesaria y conveniente, debiendo encuadrarse en un marco de corregulación, que concilie la libertad de los medios y de los actores políticos con los derechos de los ciudadanos a ser bien informados, a través de un regulador independiente del sector audiovisual -órgano en el que pone mucho énfasis la nueva Directiva europea- y de la Junta Electoral en última instancia. Esta dualidad de la corregulación es el modelo europeo más consolidado, que el Reino Unido desarrolla a través de OFCOM (The Office of Communications) y los tribunales.

La regulación por ley de los debates sería positiva porque permitiría incorporar el derecho del público a participar, como espectadores o actores, sin restar la libertad de los candidatos a intervenir o no en los mismos. La regulación, que en todo caso podría ser una parte de la obligación que tiene el audiovisual público de disponer de medios al servicio de los candidatos para acceder a la audiencia, ampliaría la diversidad, el pluralismo y la independencia. Esta regulación no interfiere en absoluto con los principios de la legislación o jurisprudencia electoral española ni europea, que tiene como misión proteger los derechos básicos sin interferir en su ejercicio.

Desde la aprobación de la vigente legislación electoral española, en 1985, y a pesar de sus sucesivas reformas, se han desarrollado cambios mucho más amplios y ambiciosos en el sistema de comunicación en general y de los medios de difusión en particular. Se amplió el sistema de medios de comunicación públicos y se multiplicó tanto el número como el impacto de los canales privados, la tecnología analógica dio paso a la digital, la convergencia e Internet diversificaron todavía más el panorama, la regulación europea reconvirtió la denominación del concepto de radiotelevisión en el de servicios audiovisuales lineales o no lineales, luego aparecen las redes sociales y las plataformas digitales y ahora llega la inteligencia artificial, smart TV, el primer apagón de la TDT, el acceso 5G y la comunicación audiovisual de la nube. Con todo eso cambiaron las formas de acceso, consumo y producción de los contenidos.

En ese contexto de cambios y mutaciones cobra importancia la realización de debates electorales, como acontecimientos de interés general, con mucha mayor relevancia que los tradicionales espacios gratuitos de propaganda electoral, la obsolescencia de las restricciones sobre la difusión de encuestas, la ausencia de regulación sobre el impacto de nuevas tecnologías o fenómenos de propaganda como las fake news y la problemática de los formatos convencionales de cobertura informativa de las campañas de los medios públicos, constreñidos por la presión de los partidos políticos y la 
autocensura del riesgo de sanciones de las Juntas electorales. La suma de todos estos factores conforma un argumento suficiente para una nueva codificación de la regulación sobre la cobertura electoral en los medios de comunicación.

\section{Referencias bibliográficas}

Anstead, N. (2015). Televised Debates in Parliamentary Democracies. Media Policy Brief, 13. London School of Economics and Political Science. https://blogs.lse.ac.uk/mediapolicyproject/

Babos, P. \& Vilagi, A. (2018). Just a Show? Effects of Televised Debates on Political Attitudes and Preferences in Slovakia. East European Politics and Societies, 32(4), 720-747.

Bachrach, P. \& Botwinick, A. (1992). Power and Empowerment. A Radical Theory of Participatory Democracy. Temple University Press.

Bruschke, J. \& Divine, L. (2017). Debunking Nixon's radio victory in the 1960 election: Reanalyzing the historical record and considering currently unexamined polling data. Social Science Journal, 54(1), 67-75.

Campo Vidal, M. (2019). El debate electoral es un antídoto contra las fake news y la posverdad. Entrevista de Chus Gómez en Diario de Pontevedra, 1/10/2019. https://www.diariodepontevedra.es/articulo/pontevedra/manuel-campo-vidal-debate-electoral-esantidoto-fake-news-posverdad/201910011746251054485.html

Campos-Freire, F., De Aguilera, M. y Rodríguez Castro, M. (2018). Impacto de las plataformas globales en la competencia mediática y los resultados de las empresas de comunicación europeas. Communication \& Society, 31(3), 223-238.

Castro, E. (2008). ¿Periodismo o propaganda? La información en los procesos electorales. Cuadernos de Periodistas. Asociación de la Prensa de Madrid. https://www.apmadrid.es/wpcontent/uploads/2008/03/doc_vapm20100514133647.pdf

Common, M. F. (2018). Facebook and Cambridge Analytica: let this be the high-water mark for impunity. LSE Business Review. http://eprints.lse.ac.uk/88964/1/businessreview-2018-03-22facebook-and-cambridge-analytica-let-this.pdf

Conde-Vázquez, E., Puentes-Rivera, I. y López-López, P.C. (2019). Electoral television debate and infotainment Elections to the Spanish Parliament. 14th Iberian Conference on Information Systems and Technologies, Coimbra (Portugal).

Congreso Debate TV (2019). Los Debates Electorales a Debate. I Congreso Internacional sobre Debates Electorales Televisados y Nuevas Formas de Comunicación Política. Organizado por el Proyecto de Investigación Debate TV, liderado por los profesores José Rúas Araujo y Ana Belén Fernández Souto, del Grupo CP2 de la Facultad de Comunicación Audiovisual de Pontevedra de la Universidad de Vigo. https://ivanpuentes5.wixsite.com/debatv2019

Díaz Arias, R. (2019). Consultas públicas sobre el futuro del audiovisual. https://teledetodos.es/index.php/blogs/item/1705-consultas-publicas-sobre-el-futuro-delaudiovisual 
DOUE (2018). Directiva (UE) 2018/1808 del Parlamento Europeo y del Consejo de noviembre de 2018 por la que se modifica la Directiva 2010/13/UE sobre la coordinación de determinadas disposiciones legales, reglamentarias y administrativas de los Estados miembros relativas a la prestación de servicios de comunicación audiovisual. https://www.boe.es/doue/2018/303/L0006900092.pdf

Fung, A. (2006). Varieties of Participation in Complex Governance. Public Administration Review, (66), 66-75.

Gallego Reguera, M. y Bernárdez Rodal, A. (2017). Influencia y repercusión mediática de los debates "cara a cara" celebrados ante las elecciones generales de 2008: José Luis Rodríguez Zapatero (PSOE) vs Mariano Rajoy (PP). Vivat Academia. Revista de Comunicación, (141). http://www.vivatacademia.net/index.php/vivat/article/view/1098

Garro, H. (2019). Conformity Voting and the Value of Public Information. Political Science Research and Methods, 7(4), 913-920.

HLEG (2018). Final report High Level Expert Group fake news and online desinformation. https://ec.europa.eu/digital-single-market/en/news/final-report-high-level-expert-group-fakenews-and-online-disinformation

House of Lords (2014). Broadcast general election debates. Select Committee on Communications 2nd Report of Session 2013-14.

https://publications.parliament.uk/pa/ld201314/ldselect/ldcomuni/171/171.pdf

Instituto RTVE (2019). RTVE ante el nuevo marco europeo para el audiovisual. http://www.rtve.es/rtve/20190220/rtve-ante-nuevo-marco-europeo/1887269.shtml

ISACA (2018). Opinión de ISACA Madrid acerca del proyecto de Ley Orgánica de Protección de Datos Personales y Garantía de los Derechos Digitales.

https://www.linkedin.com/pulse/opini\%C3\%B3n-de-isaca-madrid-acerca-del-proyecto-leyorg\%C3\%A1nica-chapter

Klein, M. \& Rosar, U. (2007). Effects of the TV-duel in the run-up to the German Federal Election 2005 on the voting decision. An empirical investigation with special regard to media influences on the winner perception and subjective expectations towards the debate performance of the candidates. Kolner Zeitschrifg fur Soziologie und Sozaialpsychologie, 59(1), 81-sgs.

López-García, G., Llorca-Abad, G., Valera-Ordaz, L. y Peris Blanes, A. (2018). Debates Electorales. ¿La última fortaleza contra la mediatización? Un estudio de caso de las elecciones generales españolas de 2015. Palabra Clave, 21(3), 772-797.

LOREG (1985). Ley Orgánica 5/1985 del Régimen Electoral General. Texto consolidado. http://www.juntaelectoralcentral.es/cs/jec/loreg/contenido?ambito=1\&ambitoModificacion=1\%20 \&annoLey=1985\&annoLeyModificacion=1985\&numeroLey=5\&numeroLeyModificacion=5\&tip $\underline{\mathrm{oLey}}=4 \&$ tipoLeyModificacion $=4$

Mani Redondo, P. (2017). Evaluación y análisis del "Media-Policy Making" de la reforma socialista del sector audiovisual y la Ley General de la Comunicación Audiovisual (2004-2011). Tesis doctoral dirigida por el profesor Carles Lloréns. Universidad Autónoma de Barcelona. 
Marqués-Pascual, J., Fondevila-Gascón, J. F., De Uribe-Gil, C. y Perelló-Sobrepere, M. (2016). Los bloques electorales en España. Una propuesta de modelo alternativo para superar el conflicto. Revista Latina de Comunicación Social, (71), 654-667. http://www.revistalatinacs.org/071/paper/1114/34es.html

Maurer, M. (2016). Nonverbale politische Kommunikation. Springer VS.

Maurer, M. \& Reinemann, C. (2003). Schröder gegen Stoiber. Nutzung, Wahrnehmung und Wirkung der TV-Duelle. Westdeutscher Verlag.

Maurer, M., Reinemann, C., Maier, J. \& Maier, M. (2007). Schröder gegen Merkel. Wahrnehmung und Wirkung des TV-Duells 2005 im Ost-West-Vergleich. VS Verlag für Sozialwissenschaften.

Mazaira-Castro, A., Rúas-Araújo, J. y Puentes-Rivera, I. (2019). Fact-Checking en los debates electorales televisados de las elecciones generales de 2015 y 2016. Revista Latina de Comunicación Social, (74), 748-766. http://www.revistalatinacs.org/074paper/1355/38es.html

MEE (2019). Consulta pública previa sobre la modificación de la Ley 7/2010, de 31 de marzo, General de la Comunicación Audiovisual. Secretaría de Estado de Avance Digital del Ministerio de Economía y Empresa.

https://avancedigital.gob.es/es-es/Participacion/Paginas/Cerradas/modificacion-ley-72010comunicacion-audiovisual.aspx

Miguel de Bustos, J. C., e Izquierdo-Castillo, J. (2019). ¿Quién controlará la Comunicación? El impacto de los GAFAM sobre las industrias mediáticas en el entorno de la economía digital. Revista Latina de Comunicación Social, (74), 803-821.

http://www.revistalatinacs.org/074paper/1358/41es.html

Navarro Marchante, V. (2019). Los debates electorales en televisión: una necesaria revisión de su regulación. Revista Española de Derecho Constitucional, 39(116), 110.

Novos Medios (2019). Participación de profesores de Universidades españolas y expertos en TDT en la Consulta Pública sobre la modificación de la Ley General del Audiovisual 7/2010. http://novosmedios.gal/consulta-publica-ley-audiovisual-2010/

Perales, P. (2018). Nueva Directiva de Servicios de comunicación audiovisual. https://teledetodos.es/index.php/blogs/item/1689-nueva-directiva-de-servicios-de-comunicacion$\underline{\text { audiovisual }}$

Saavedra Llamas, M. \& Rodríguez Fernández, L. (2018). Spanish Television Channels and the 34-J Election Debate: Programming Strategies and Social Audience. Fonseca, (17), 125-136.

Schneble, Ch. O., Elger, B. S. \& Shaw, S. (2018). The Cambridge Analytica affair and Internetmediated research. EMBO. https://www.embopress.org/doi/pdf/10.15252/embr.201846579

Tambini, D. (2018). Study on the use of internet in electoral campaigns. Council of Europe study, DGI (2017) 11. Prepared by the committee of experts on media pluralism and transparency of media ownership (MSI-MED). https://rm.coe.int/use-of-internet-in-electoral-campaigns$\underline{16807 \mathrm{c} 0 \mathrm{e} 24}$ 
VV AA. (2012). Periodismo político en España: concepciones, tensiones y elecciones. Cuadernos Artesanos de Comunicación Latina, (33).

http://www.revistalatinacs.org/067/cuadernos/artesanos.html\#33

Zunger, J. (2018). Computer science faces an ethics crisis. The Cambridge Analytica sacandal proves it. Boston Globe. https://delaat.net/ecis/Computer-science-faces-an-ethics-crisis-The-BostonGlobe.pdf

\section{AUTORES:}

\section{Francisco Campos-Freire}

Periodista y catedrático de Periodismo en la Facultad de Ciencias de la Comunicación de la Universidad de Santiago de Compostela (España). Licenciado en Ciencias de la Información por la Universidad Complutense de Madrid, Doctor por la Universidad de Santiago de Compostela, es también MBA en Dirección y Gestión de Empresas Industriales y de Servicios, Experto Europeo certificado en Gestión de Responsabilidad Social Corporativa y de la Calidad certificado por European Organization for Quality (EOQ) y Asociación Española de la Calidad (AEC). En el ámbito periodístico empezó su carrera profesional como delegado del rotativo regional La Voz de Galicia en Lugo (1979-1985), subdirector y director del diario La Región de Orense (1985-1990), directorgerente de Agencia Gallega de Noticias (AGN) en 1990-94, director general de la Compañía de Radio Televisión de Galicia desde 1994 a 200 y presidente de la Federación de Organismos de Radio y Televisión Autonómica de España (FORTA) en los años 1994, 1997, 2001 y 2004. Como académico ha publicado cerca de un centenar de artículos de investigación y una docena de libros, entre ellos los siguientes títulos en los últimos años: "Gestión de nuevas empresas periodísticas" (2008), "El cambio mediático" (2009), "El nuevo escenario mediático" (2010), "Gobernanza y comunicación en Europa" (2011) y "La gestión e investigación de las redes sociales digitales" (Cuadernos Artesanos de Comunicación, 2013).

francisco.campos@usc.es

Índice H: 14

Orcid ID: http://orcid.org/0000-0003-1831-1838

Google Scholar: https://scholar.google.es/citations?user=feJKfd8AAAAJ\&hl=es

\section{Marta Rodríguez-Castro}

Graduada en Comunicación Audiovisual por la Universidad de Santiago de Compostela (USC) y máster en Investigación aplicada a medios de comunicación por la Universidad Carlos III de Madrid. Actualmente es doctoranda en Comunicación e información contemporánea en la USC, donde es contratada FPU (FPU16/05234) e investigadora del grupo Novos Medios. Su área de investigación está vinculada a los medios de comunicación públicos europeos, prestando especial atención a su gobernanza, regulación y financiación. Su tesis doctoral aborda las pruebas de valor público europeas, así como los medios de proximidad.

m.rodriguez.castro@usc.es

Índice H: 2

Orcid ID: https://orcid.org/0000-0002-1399-9154

Google Scholar: $\underline{\text { https://scholar.google.es/citations?user=E0tEeR0AAAAJ\&hl=es }}$

\section{Alejandro Gesto-Louro}

Alejandro Gesto-Louro es graduado en Periodismo por la Universidad de Santiago de Compostela (España) con el Trabajo de Fin de Grado titulado Evolución de la financiación de los servicios audiovisuales públicos europeos (2010-2017). Estudios en Derecho por la Universidad Autónoma de 
Madrid (2014-2016). Actualmente, cursa el Máster Universitario en Periodismo y Comunicación: Nuevas Tendencias en Producción, Gestión y Difusión del Conocimiento de la Facultad de Ciencias de la Comunicación de la Universidad de Santiago de Compostela e inicia su actividad como investigador, con especial interés por la financiación, la regulación y la innovación de los medios públicos europeos.

alejandrogesto@gmail.com

Orcid ID: https://orcid.org/0000-0002-4079-0989 